\title{
Principais aspectos fisiopatológicos e clínicos presentes no Diabetes mellitus tipo I
}

\section{(autoimune)}

\author{
Main pathophysiological and clinical aspects present in type I Diabetes mellitus (autoimmune) \\ Principales aspectos fisiopatológicos y clínicos presentes en la Diabetes mellitus tipo I (autoinmune)
}

Recebido: 14/10/2021 | Revisado: 20/10/2021 | Aceito: 26/10/2021 | Publicado: 29/10/2021

Bianca Barros da Costa

ORCID: https://orcid.org/0000-0001-8962-2639 Universidade Federal do Rio de Janeiro, Brasil

E-mail: costa.biancab@gmail.com

Thamyris Almeida Moreira

ORCID: https://orcid.org/0000-0003-3718-7131

Universidade Federal do Rio de Janeiro, Brasil

E-mail: thamyris.pharma@gmail.com

\begin{abstract}
Resumo
O Diabetes mellitus (DM) é definido como uma doença crônica não transmissível, caracterizada pela presença de uma hiperglicemia crônica. Pode ser subdividida em três tipos principais: DM tipo 1 (autoimune), DM tipo 2 (resistente à insulina) e o DM gestacional. O DM tem se tornado um problema de saúde pública mundial, por isso há necessidade de estudos sobre essa doença, no intuito de melhorar o acesso à informação, pesquisa e qualidade de vida dos indivíduos. Sendo assim, este trabalho teve por objetivo descrever as principais características fisiopatológicas associadas ao DM tipo 1. A metodologia utilizada foi baseada na procura de trabalhos científicos, nas bases de dados Scielo, Lilacs, BVS, Pumed e Medline. No caso do DM tipo 1, sua principal ocorrência é devido à uma reação autoimune, gerada pela produção equivocada de anticorpos que "atacam" as células beta pancreáticas descontrolando, assim, a secreção de insulina e, consequentemente, a absorção de glicose pelas células, gerando a hiperglicemia. O DM tipo 1 tem sua origem de forma multifatorial, envolvendo características genéticas, mas também sofrendo influência de fatores ambientais. O diagnóstico clínico-laboratorial do DM tipo 1 é feita pela analise de características hereditárias bem como a sintomatologia presente, incluindo dados laboratoriais da glicemia do paciente, do percentual de HbAlc e de marcadores imunológicos específicos. Portanto, fica claro que a patogênese do DM tipo 1 envolve uma complexa rede de alterações fisiológicas e bioquímicas decorrentes do estado de hiperglicemia crônica em que o organismo se encontra devido à destruição das células beta pancreáticas.
\end{abstract}

Palavras-chave: Diabetes mellitus tipo 1; Fisiopatologia do diabetes; Complicações do diabetes; Diagnóstico do diabetes.

\begin{abstract}
Diabetes mellitus (DM) is defined as a chronic, non-communicable disease characterized by the presence of chronic hyperglycemia. It can be subdivided into three main types: type 1 DM (autoimmune), type 2 DM (insulin resistant) and gestational DM. DM has become a global public health problem, so there is a need for studies on this disease, in order to improve access to information, research and quality of life for individuals. Therefore, this study aimed to describe the main pathophysiological characteristics associated with type $1 \mathrm{DM}$. The methodology used was based on the search for scientific papers in the Scielo, Lilacs, BVS, Pumed and Medline databases. In the case of type 1 DM, its main occurrence is due to an autoimmune reaction, generated by the mistaken production of antibodies that "attack" the pancreatic beta cells, thus disrupting the secretion of insulin and, consequently, the absorption of glucose by the cells, generating hyperglycemia. Type $1 \mathrm{DM}$ has its origin in a multifactorial way, involving genetic characteristics, but also being influenced by environmental factors. The clinical and laboratory diagnosis of type 1 DM is made through the analysis of hereditary characteristics as well as the present symptoms, including laboratory data on the patient's blood glucose, the percentage of HbA1c and specific immunological markers. Therefore, it is clear that the pathogenesis of type 1 DM involves a complex network of physiological and biochemical alterations resulting from the state of chronic hyperglycemia in which the organism finds itself due to the destruction of pancreatic beta cells.
\end{abstract}

Keywords: Type 1 Diabetes mellitus; Diabetes Pathophysiology; Diabetes complications; Diagnosis of diabetes.

\section{Resumen}

La Diabetes mellitus (DM) se define como una enfermedad crónica no transmisible, caracterizada por la presencia de hiperglucemia crónica. Se puede subdividir en tres tipos principales: DM tipo 1 (autoinmune), DM tipo 2 (resistente a la insulina) y DM gestacional. La DM se ha convertido en un problema de salud pública mundial, por lo que es necesario realizar estudios sobre esta enfermedad, con el fin de mejorar el acceso a la información, la investigación y 
la calidad de vida de las personas. Por tanto, este estudio tuvo como objetivo describir las principales características fisiopatológicas asociadas a la DM tipo 1. La metodología utilizada se basó en la búsqueda de artículos científicos en las bases de datos Scielo, Lilacs, BVS, Pumed y Medline. En el caso de la DM tipo 1, su principal aparición se debe a una reacción autoinmune, generada por la producción errónea de anticuerpos que "atacan" las células beta pancreáticas, interrumpiendo así la secreción de insulina y, en consecuencia, la absorción de glucosa por parte de la células, generando hiperglucemia. La DM tipo 1 tiene su origen de forma multifactorial, involucrando características genéticas, pero también siendo influenciada por factores ambientales. El diagnóstico clínico y de laboratorio de la DM tipo 1 se realiza mediante el análisis de las características hereditarias así como de la sintomatología presente, incluyendo datos de laboratorio sobre la glucemia del paciente, el porcentaje de HbA1c y marcadores inmunológicos específicos. Por tanto, es evidente que la patogenia de la DM tipo 1 implica una compleja red de alteraciones fisiológicas y bioquímicas derivadas del estado de hiperglucemia crónica en el que se encuentra el organismo debido a la destrucción de las células beta pancreáticas.

Palabras clave: Diabetes mellitus tipo 1; Fisiopatología de la diabetes; Complicaciones de la diabetes; Diagnóstico de diabetes.

\section{Introdução}

O Diabetes mellitus (DM), segundo a Organização Mundial da Saúde (OMS), é definido como uma doença crônica não transmissível (DCNT), caracterizada pelo aumento da glicose plasmática, devido à defeitos na secreção e/ou ação da insulina produzida pelo pâncreas (OMS, 1999; International Diabetes Federation - IDF, 2019).

Essa situação fisiopatológica dificulta a entrada da glicose em determinadas células (insulinodependentes) fazendo com que haja a chamada hiperglicemia crônica, que gera complicações graves, como alterações macro e microvasculares bem como alguns distúrbios metabólicos, frequentemente associadas à disfunção ou falência de órgãos importantes, principalmente a retina dos olhos, os rins, coração e nervos. Por esse motivo, a DM necessita de cuidados constantes e um diagnóstico precoce para redução dos danos à saúde do indivíduo (Ferreira et al., 2011; American Diabetes Association - ADA, 2015; IDF, 2019).

A DM é considerada a DCNT mais prevalente no mundo, estimando-se mais de 463 milhões de adultos com diabetes, de acordo com dados de 2019 da Federação Internacional de Diabetes (IDF), sendo que cerca de 50\% destes indivíduos não sabem que possuem a doença. Estudos recentes mostram uma projeção de aproximadamente 700 milhões de pessoas com diabetes em 2045 e, por esse motivo, é considerada um problema de saúde mundial. O Brasil se encontra na quinta posição dentre os países com maior número de casos de diabetes em adultos com faixa etária de 20-79 anos, como mostra a figura 1 (Flor \& Campos, 2017; IDF, 2019).

Figura 1: Top 10 dos países com maior número de casos de diabetes em adultos com idade entre 20-79 anos.

\begin{tabular}{|c|c|c|c|c|c|c|}
\hline \multirow[b]{2}{*}{ Rank } & \multicolumn{2}{|c|}{2019} & \multicolumn{2}{|c|}{2030} & \multicolumn{2}{|c|}{2045} \\
\hline & $\begin{array}{l}\text { Country or } \\
\text { territory }\end{array}$ & $\begin{array}{l}\text { No. of people } \\
\text { w diabetes } \\
\text { (millions) }\end{array}$ & $\begin{array}{l}\text { Country or } \\
\text { territory }\end{array}$ & $\begin{array}{l}\text { No. of people } \\
\text { w diabetes } \\
\text { (millions) }\end{array}$ & $\begin{array}{l}\text { Country or } \\
\text { territory }\end{array}$ & $\begin{array}{l}\text { No. of people } \\
\text { w diabetes } \\
\text { (millions) }\end{array}$ \\
\hline 1 & China & 116.4 & China & 140.5 & China & 147.2 \\
\hline 2 & India & 77.0 & India & 101.0 & India & 134.2 \\
\hline 3 & $\begin{array}{l}\text { United States of } \\
\text { America }\end{array}$ & 31.0 & $\begin{array}{l}\text { United States of } \\
\text { America }\end{array}$ & 34.4 & Pakistan & 37.1 \\
\hline 4 & Pakistan & 19.4 & Pakistan & 26.2 & $\begin{array}{c}\text { United States of } \\
\text { America }\end{array}$ & 36.0 \\
\hline 5 & Brazil & 16.8 & Brazil & 21.5 & Brazil & 26.0 \\
\hline 6 & Mexico & 12.8 & Mexico & 17.2 & Mexico & 22.3 \\
\hline 7 & Indonesia & 10.7 & Indonesia & 13.7 & Egypt & 16.9 \\
\hline 8 & Germany & 9.5 & Egypt & 11.9 & Indonesia & 16.6 \\
\hline 9 & Egypt & 8.9 & Bangladesh & 11.4 & Bangladesh & 15.0 \\
\hline 10 & Bangladesh & 8.4 & Germany & 10.1 & Turkey & 10.4 \\
\hline
\end{tabular}

Fonte: International Diabetes Federation (IDF) (2019). 
De uma forma geral, a DM pode ser classificada em 3 subtipos principais e mais conhecidos: $1^{\circ}$ ) DM tipo 1: provocada por reações autoimunes; $2^{\circ}$ ) DM tipo 2: hiperglicemia gerada por uma resistência à insulina; $3^{\circ}$ ) DM gestacional: quaro de hiperglicemia durante a gravidez que, geralmente, desaparece após o parto (IDF, 2019).

O DM tipo 1 é considerado de origem autoimune, ocorrendo devido à presença de anticorpos que atacam as células beta-pancreáticas, gerando uma destruição parcial ou total destas células, assim a produção/secreção de insulina fica prejudicada, levando então aos sinais e sintomas característicos da doença (ADA, 2015; Neves et al., 2017; IDF, 2019).

Com o aumento expressivo dos números de indivíduos com DM no mundo, faz-se necessário que se aumentem os estudos relativos à doença, para que haja uma melhoria nas informações disponíveis aos pacientes, à comunidade e aos profissionais da saúde. Dessa forma todo o conhecimento adquirido trará, também, uma melhoria no padrão de vida dos pacientes diabéticos, assim como nos cuidados com à saúde da população em geral, na tentativa de redução do número de complicações associadas ao DM e da morbimortalidade.

Sendo assim, o objetivo deste trabalho é trazer uma revisão da literatura, descrevendo as principais características fisiopatológicas associadas ao DM tipo 1, citando fatores fisiológicos gerais, bem como a etiopatogenia, diagnósticos clínicolaboratoriais atualmente empregados e possíveis complicações.

\section{Metodologia}

Para a elaboração deste estudo, realizou-se uma revisão narrativa da literatura, com caráter descritivo-qualitativo, utilizando-se trabalhos científicos publicados nas bases de dados Scientific Electronic Library Online (SciELO); Literatura Latino-Americana e do Caribe em Ciências da Saúde-LILACS, Biblioteca Virtual de Saúde (BVS), Medline e Pubmed.

A busca e análise dos trabalhos foi realizada entre março de 2020 e agosto de 2021, analisando-se os títulos e resumos, optando-se pelos que realmente se enquadravam na temática e nos critérios de inclusão (Estrela, 2018). Foram utilizados os descritores: "diabetes mellitus tipo 1", "alterações fisiopatológicas do diabetes", "complicações do diabetes mellitus", "diagnóstico do diabetes mellitus".

Como critérios de inclusão foram utilizados artigos, publicações científicas e teses, nas línguas portuguesa e inglesa, entre os anos de 2000 e 2020. Foram excluídos trabalhos que não se enquadravam no tema, bem como estudos de campo restritos a regiões específicas.

\section{Resultados e Discussão}

\subsection{Fisiologia do Pâncreas endócrino - Secreção e ação da insulina}

O pâncreas é um órgão lobulado que possui uma alta vascularização, estando situado na cavidade abdominal com adjacências na porção esquerda do baço e na porção direita do duodeno, sendo ligada a este órgão por tecido conjuntivo denso fibroso (Lopes, 2017). É uma glândula fundamental na digestão de alimentos e na regulação do metabolismo, sendo didaticamente dividido em exócrino (ou exógeno), responsável por sintetizar e secretar enzimas digestivas, e o endócrino (ou endógeno) que apresenta três subpopulações principais, de células especializadas (alfa, beta e delta) nas chamadas ilhotas de Langerhans, produzindo e secretando os hormônios insulina, glucagon e somatostatina, respectivamente (Montenegro et al., 2016).

A insulina é um peptídeo, geralmente liberado em períodos absortivos, produzido pelas células beta-pancreáticas em resposta à elevação da glicose plasmática, exercendo seus efeitos principalmente no musculo esquelético, cardíaco, no fígado e tecido adiposo. Ao se ligar na porção alfa do seu respectivo receptor (IRS) uma rápida mudança conformacional induz o aumento da captação da glicose circulante para dentro das células de tecidos insulinodependentes, através da mobilização de receptores específicos de glicose (GLUT) para as membranas celulares (Nolasco \& Martins, 2017). 
A taxa de secreção de insulina depende do grau de estímulos excitatórios e inibitórios que as células beta-pancreáticas recebem. A concentração sanguínea de glicose, como dito anteriormente, é a causa fundamental de estímulo já que a medida que a glicemia se eleva (período pós-prandial), as células betas controlam diretamente o aumento da produção e secreção de insulina adicional para aumentar, também, a captação tecidual de glicose e reduzir os níveis séricos. Sendo assim, qualquer alteração na fisiologia das células beta-pancreáticas, gera um distúrbio na secreção de insulina, consequentemente, influenciando na glicemia do indivíduo (Mitchell, 2012; Silva \& Rego, 2021).

\subsection{Fisiopatologia e etiopatogenia do DM tipo 1}

O DM tipo 1 é uma DCNT caracterizada pela presença de hiperglicemia em indivíduos cuja secreção de insulina está sendo prejudicada diretamente, sendo considerada de origem autoimune devido à produção equivocada de anticorpos que atacam as células beta-pancreáticas, gerando então a destruição parcial ou total dessas céluas. Geralmente o DM tipo 1 é detectado quando há destruição de mais de $80 \%$ das células. Consequentemente, determinados tecidos se tornam incapazes de captar glicose de forma eficiente, assim, esta molécula tende a se acumular na circulação sanguínea, gerando a chamada hiperglicemia crônica (van Belle et al., 2011; ADA, 2015; IDF, 2019)

A natureza autoimune do DM tipo 1 pode ser evidenciada pela presença de células autorreativas e autoanticorpos, que podem estar presentes nos indivíduos por anos, e até por décadas, antes mesmo do desenvolvimento da doença. No entanto, o conhecimento sobre os mecanismos atuantes que desencadeiam a morte das células pancreáticas e a inflamação local ainda é muito limitado. Acredita-se que as células imunes presentes nas ilhotas de pacientes com DM tipo 1 sejam, majoritariamente, linfócitos TCD8, TCD4, células B e macrófagos. Assim, percebe-se a ativação tanto da resposta imune inata quanto da adaptativa, culminando na morte das células beta-pancreáticas (Nguyen et al., 2013; Guay \& Regazzi, 2013; Cavalcante et al., 2020).

Apesar de o DM tipo 1 um ser uma doença caracterizada pela presença da carga genética para desencadear as reações autoimunes, já é descrito em literatura um caráter multifatorial, com a influência de fatores ambientais e a exposição a infecções virais (Atkinson et al., 2014; IDF, 2019). Dentre os fatores genéticos envolvidos, podemos destacar os relacionados com o Major histocompatibility complex (MHC) no cromossoma 6p21, o gene da insulina na região 1lp15, o gene CTLA-4 (cytotoxic T lymphocyte associated-4) no cromossoma 2q33, o MIC-A (MHC I-gene related A), os genes codificantes de interleucinas (IL-2, IL-21, IL-6, IL-10, IL-9, IL-20 e IL-27) e os genes HLA - apresentadores de antígenos - no cromossoma 6, responsáveis por mais de 50\% do risco de desenvolvimento do DM tipo 1 (Delli \& Lernmark, 2016; Neves et al., 2017).

Além da influência genética, sabe-se que os fatores ambientais possuem um papel relevante na agressão imune presente no DM tipo 1. O desenvolvimento da microbiota na criança, normalmente, acontece nos primeiros meses de vida, sendo assim a presença de determinadas proteínas alimentares, principalmente $\beta$-lactoglobulina, a $\beta$-caseína e a albumina bovina, e de alguns enterovírus podem levar ao desenvolvimento de autoimunidade que leva à destruição das células beta pancreáticas tendo por consequência um estresse metabólico e um processo inflamatório local, prejudicando então o funcionamento dessas células e levando ao DM tipo 1 (Neves et al., 2017).

\subsection{Diagnósticos clínico - laboratorial do DM tipo 1}

O DM tipo 1 geralmente apresenta sinais e sintomas ainda na infância/juventude dos indivíduos, através de sinais clássicos como: polidipsia, poliúria e emagrecimento sem explicação, podendo ainda apresentar irritabilidade, desidratação e a descompensação em cetoacidose (SBD, 2015). Pelo fato de ter características de hereditariedade, existe um trabalho de anamnese e investigação de histórico familiar, devido à predisposição genética para gerar uma disfunção autoimune nas células beta pancreáticas (Barreto, 2016). 
Para o diagnóstico laboratorial do DM tipo 1, existem alguns parâmetros que precisam ser avaliados e, na maioria das vezes, seguem as normas selecionadas pela OMS, ADA e a Sociedade Brasileira de Diabetes (SBD). De acordo com essas entidades, os parâmetros adotados para o diagnóstico ficam pré-estabelecido da seguinte forma: Glicemia em jejum $\geq 126$ $\mathrm{mg} / \mathrm{dL}$ e glicemia aleatória (coletada em qualquer hora do dia) $\geq 200 \mathrm{mg} / \mathrm{dL}$, em pacientes com sintomas característicos do DM. Na maioria dos casos, não é necessário utilizar o Teste Oral de Tolerancia à glicose (TOTG), no entanto quando houver confusão clínica com o DM tipo 2, faz-se a glicemia após a ingestão oral (sobrecarga) de 75 gramas de glicose que deve estar abaixo de $200 \mathrm{mg} / \mathrm{dL}$ para ser considerado normal. Esses critérios foram estabelecidos baseados na observação de que valores acima destes anteriormente citados estão fortemente relacionados com o aumento da incidência de complicações microvasculares (ADA, 2015; Silva \& Rego, 2021).

Antigamente a hemoglobina glicada (fração HbA1c) era utilizada somente como forma de monitoramento dos pacientes diabéticos. No entanto, recentemente, seu uso também foi aprovado pela ADA e pela OMS para o diagnóstico do DM. Assim, os valores são considerados normais entre 4,5 - 5,6\%, pré-diabetes entre 5,7 - 6,4\% e valores $\geq 6,5 \%$ são considerados como um possível diagnóstico de DM, sendo, as vezes, necessária a repetição dos exames para confirmação do diagnóstico de forma segura e precisa (Forouhi \& Wareham, 2014; Barreto, 2016; Oliveira et al., 2020).

Um outro marcador importante é o índice HOMA-IR (Homeostasis Model Assessment for Insulin Resistance), utilizado frequentemente por possuir a vantagem de ser calculado através de uma única amostra de sangue. Esse índice é calculado pela fórmula [(FPI $\mu \mathrm{U} / \mathrm{mL}$ x FPG mmol/L) / 22,5], onde se compara a quantidade de insulina no plasma em jejum (FPI) com a quantidade de glicose no plasma em jejum (FPG) e valor de resistência da população descrita como de referência (SBD, 2015, Fonseca et al., 2018).

No DM tipo 1 é comum serem encontrados alguns marcadores mais específicos que requerem uma investigação laboratorial mais aprofundada para confirmação da etiologia autoimune, são eles: dosagem de Peptídeo C (que podem estar em níveis reduzidos), de células $\mathrm{T}$ autorreativas, autoanticorpos para a insulina (IAAs), para as células das ilhotas de Langerhans (ICA512), para a enzima descarboxilase do ácido glutâmico (GAD65), para o transportador de zinco específico de células beta (ZnT8) e para as fosfatases de tirosina (IA-2 e IA-2ß) (Guay \& Regazzi, 2013; ADA, 2015; Silva \& Rego, 2021).

Cerca de 85 e $90 \%$ dos indivíduos com DM tipo 1 apresentam dois ou mais desses marcadores e no caso específico desse tipo de DM, a destruição das células beta pancreáticas pode levar meses ou anos, sendo clinicamente detectada após a destruição de mais de 80\% dessas células (van Belle, 2011; Silva \& Rego, 2021).

\subsection{Complicações do DM não tratado}

O DM quando não tratado pode aumentar o risco de morte precoce. A IDF estimou que em 2019 aproximadamente 4,2 milhões de adultos morreriam em decorrência do diabetes e de suas complicações (IDF, 2019).

A maioria das complicações advindas do DM é vista em pessoas que, por algum motivo, desconheciam que possuíam a doença e, por isso, não a tratavam corretamente. No entanto, a maneira como os indivíduos diabéticos, cientes da sua doença, procuram basear seu estilo de vida, pode influenciar diretamente no aparecimento de complicações graves ligadas à hiperglicemia. Fatores como sedentarismo, alimentação desregulada e a forma de controle individual da glicemia podem ser determinantes no surgimento de complicações agudas como: cetoacidose diabética - mais comum no DM tipo 1; Coma hiperosmolar - mais prevalente no DM tipo 2; e hipoglicemia - com níveis glicêmicos abaixo de 70mg/dL, gerando sintomas de confusão mental, fraqueza, tremores, dentre outros que podem acarretar em perda de consciência, coma e até mesmo levar à morte, em casos mais graves (IDF, 2019; Fonseca \& Rached, 2019).

A severidade do DM pode ser amplificada no caso do surgimento de complicações crônicas devido a alterações micro e macrovasculares. No primeiro caso, pode ocorrer o desenvolvimento de: 
a) Neuropatia periférica - maior prevalência dentre as disfunções crônicas, chegando a afetar 40\% dos indivíduos com DM, podendo afetar sistemas importantes como o cardiovascular, digestivo e urogenital (Nunes et al., 2021);

b) Retinopatia - afeta gravemente o globo ocular, sendo a principal causa de cegueira irreversível (Fonseca \& Rached, 2019);

c) Nefropatia - gera uma alteração no processo de filtração glomerular, sendo a principal causa de insuficiência renal (Pasqualoto et al., 2012; Silva, Silva \& Romão, 2020);

d) Pé diabético - é maior causa de internações, sendo caracterizada por lesões nós pés que não se cicatrizam em decorrência das alterações vasculares periféricas, podendo levar á formação de úlceras, que são consideradas a "porta de entrada" para necrose e a amputação dos membros (Mantovani et al., 2013; Gois \& Chaves, 2020).

Já no caso das alterações macrovasculares, pode ocorrer o desenvolvimento de doença arterial coronariana, doença cerebrovascular e vascular periférica, que podem levar o indivíduo à morte. Além das citadas anteriormente, existem outras complicações que já foram diretamente relacionadas com o DM, tais como hipertensão arterial, dislipidemias, depressão, doença periodontal, escleredema de Buschke e gastroenteropatia diabética (Fonseca \& Rached, 2019).

\section{Conclusão}

O DM tipo 1 possui uma origem multifatorial que envolve uma complexa rede de genes e alterações fisiológicas e bioquímicas, desencadeando reações autoimunes, gerando a produção de anticorpos específicos que culminam na destruição das células beta pancreáticas, prejudicando assim a produção e secreção de insulina. Com isso, há uma redução da absorção de glicose pelas células, resultando num quadro de hiperglicemia crônica.

O diagnóstico do DM tipo 1 envolve a avaliação do histórico familiar bem como da sintomatologia característica dos indivíduos diabéticos, que apresentam poliúria, polidipsia, emagrecimento e, geralmente, descompensação por cetoacidose diabética. Por esse motivo, é necessário um diagnóstico associado à avaliação laboratorial, através, principalmente, da análise da glicemia em jejum, da glicemia aleatória e por sobrecarga, da dosagem de HbA1c, do índice de HOMA-IR, de peptídeo C e dos marcadores imunológicos específicos.

A existência e persistência de estado hiperglicêmico não controlado é o fator relevante no desencadeamento de complicações macrovasculares, microvasculares e dos distúrbios metabólicos no indivíduo diabético, que podem gerar quadros de cegueira, necrose com amputação de membros e até mesmo a morte.

Portanto, fica clara a complexidade dos mecanismos envolvidos na fisiopatologia do DM tipo 1 e do nível de gravidade da influência que as alterações fisiológicas, imunológicas e bioquímicas, decorrentes da hiperglicemia crônica, têm no estado e qualidade de vida dos indivíduos. Desse modo, trabalhos futuros podem trazer a aplicação de novos biomarcadores para diagnóstico e acompanhamento do DM bem como informações mais detalhadas sobre o mecanismo imunológico envolvido no processo da hiperglicemia, ressaltando a presença da inflamação e o estresse oxidativo gerado, levando em consideração também algumas populações especiais como gestantes, idosos e crianças.

\section{Referências}

American Diabetes Association - ADA. (2015) Classification and diagnosis of diabetes. Diabetes Care 38(Suppl):S8-S16.

Atkinson, M. A, Eisenbarth, G. S., \& Michels, A. W. (2014) Type 1 Diabetes. Lancet. 383: 69-82.

Barreto, F. K. A. (2016) Atividade antihiperglicêmica e antioxidante da lectina de bryothamnion seaforthii em ratos com diabetes induzido por estreptozotocina. Tese de doutorado. Faculdade de medicina - Departamento de patologia e medicina legal. Universidade Federal do Ceará.

Cavalcante, G.L. et al. (2020) Perfil farmacoepidemiológico de pacientes com diabetes mellitus tipo 1. Research, Society and Development, 9(5). http://dx.doi.org/10.33448/rsd-v9i5.3361. 
Concannon, P. et al. (2008) A human type 1 diabetes susceptibility locus maps to chromosome 21q22.3. Diabetes. 57(10): 2858.

Delli, J. \& Lernmark, A. (2016) Etiology, Pathogenesis, Prediction, and Prevention. In: Ahmed. Endocrinology (DeGroot), 7 th edition, p. 672 - 690.

Estrela, C. (2018). Metodologia Científica: Ciência, Ensino, Pesquisa. Editora Artes Médicas.

Ferreira, L. T. et al. (2011) Diabetes mellitus: hyperglycemia and its chronic complications. Arq. Bras. de Ciências da Saúde, 36(3), 182-8.

Flor, L. S. \& Campos, M. R. (2017) Prevalência de diabetes mellitus e fatores associados na população adulta brasileira: evidências de um inquérito de base populacional. Rev. Bras. Epidemiol., 20(1), 16-29.

Fonseca, E. J. N. C. et al. (2018) Metabolic Syndrome and Insulin Resistance by HOMA-IR in Menopause. Int. J. Cardiovasc. Sci. 31 (3). https://doi.org/10.5935/2359-4802.20180009.

Fonseca, K. P. \& Rached, C. D. A. (2019) Complicações do Diabetes Mellitus - Complications of diabetes mellitus. International Journal of Health Management $-\mathrm{Ed} \mathrm{n}^{\circ} 1$.

Gois, J. P. S. \& Chaves, A. S. C. (2020) Pé diabético: avaliação dos fatores de risco relacionados a amputações maiores e menores. Revista Eletrônica Acervo Saúde, 12 (1). https://doi.org/10.25248/reas.e1484.2020.

Guay, C. \& Regazzi, R. (2013) Circulating microRNAs as novel biomarkers for diabetes mellitus. Nat Rev Endocrinol 9(9):513-521.

International Diabetes Federation - IDF. (2019) IDF diabetes atlas. (9th ed.) www.diabetesatlas.org.

Lopes, T. C. A. (2017) Anomalias congénitas do pâncreas - estado da arte. Faculdade de Medicina, Dissertação de mestrado - Universidade de Coimbra Portugal.

Mantovani, A. M. et al. (2013) Estudo comparativo das representações sociais sobre diabetes mellitus e pé diabético. Cad. Saúde Pública. 29(12), 2427-2435.

Mitchell et al. (2012) Robbins \& Cotran: Fundamentos de patologia. (8a ed.), Saunders Elsevier.

Montenegro, R., Chaves, M. \& Fernandes, V. (2016) Fisiologia pancreática: pâncreas endócrino. Ameneiros Rodriguez.

Neves, C. et al. (2017) Diabetes Mellitus tipo 1. Revista Portuguesa de Diabetes. 12 (4): 159-167.

Nolasco, E. L. \& Martins, J. O. (2017) Insulina regula a translocação nuclear de NFkB p65, inflamação e morte celular em modelo experimental de sepse em camundongos diabéticos. Tese de doutorado. Universidade de São Paulo. http://www.teses.usp.br/teses/disponiveis/9/9142/tde-07072017-182830/.

Nguyen, C. et al. (2013) Definition of high-risk type 1 diabetes HLA-DR and HLA-DQ types using only three single nucleotide polymorphisms. Diabetes 62(6):2135-2140.

Nunes, A. C. et al. (2021) Estudo piloto da prevalência de neuropatia periférica em adultos diagnosticados com Diabetes mellitustipo 2 na Atenção Primária em saúde de Divinópolis -Brasil. Research, Society and Development, 10(11). https://doi.org/10.33448/rsd-v10i11.19568.

Oliveira, E. et al. (2020) Diabetes mellitus: avaliação e controle através de exames laboratoriais em umserviço privado de Teresina, Piauí, Brasil. Research, Society and Development, 9(2). http://dx.doi.org/10.33448/rsd-v9i2.2063.

Organização Mundial da Saúde - OMS. (1999) Definition, diagnosis and classification of diabetes mellitus and its complications: report of a WHO consultation. Part 1: diagnosis and classification of diabetes mellitus. Genebra. http://goo.gl/iZu3mU.

Pasqualotto, K. R, Alberton, D. \& Frigeri, H. R. (2012) Diabetes mellitus e Complicações. J. Biotec. Biodivers, 3(4), $134-145$.

Silva, M. C. G. \& Rêgo, J. F. (2021) Uma alternativa no diagnóstico e monitoramento de Diabetes Mellitus a detecção via biomarcadores:uma revisão sistemática. Research, Society and Development, 10(10). https://doi.org/10.33448/rsd-v10i10.18736.

Silva, A. P. P, Silva, A. R. \& Romão, J. A. (2020) Nefropatia diabética: uma revisão integrativa da literatura. Research, Society and Development, 9(10). http://dx.doi.org/10.33448/rsd-v9i10.9082.

Sociedade Brasileira de Diabetes - SBD. (2015) Diretrizes da Sociedade Brasileira de Diabetes: 2014-2015.AC Farmacêutica. http://www.diabetes.org.br/images/2015/area-restrita/diretrizes-sbd-2015.pdf.

Van Belle, T. L., Coppieters, K. T. \&Von Herrath, M. G. (2011) Type 1 diabetes: etiology, immunology, and therapeutic strategies. Physiol Rev 91(1):79-118. 